thétique bouchère, si ce dernier classement demeure inchangé, en croisement avec des mâles Limousins et Blonds d'Aquitaine, en Charolais toutefois les races laitières de grand format comme la Pie-Rouge de l'Est, la Brune des Alpes et la Normande permettent une meilleure expression du potentiel de croissance de ces mâles.

Ces résultats indiquent notamment que la valorisation des races comme femelles allaitantes support d'un croisement avec des races à viande, implique la recherche d'une complémentarité entre les composantes maternelles des premières et directes des secondes, en vue d'obtenir un équilibre optimum entre le format, les caractères de croissance (potentiel de croissance, production laitière) et la conformation.

\title{
APTITUDE A LA GÉMELLITTÉ DES RACES A VIANDE FRANÇAISES : OBSERVATIONS EN ÉLEVAGE ET CONSTITUTION D'UN TROUPEAU DE SÉLECTION
}

\author{
F. Ménissier, J. Frebling. - Département de Génétique animale, C. N. R. Z., 78350, Jouy en \\ Josas (France).
}

Du fait des récents progrès dans les techniques de reproduction et de l'existence d'une variabilité d'origine génétique de la fréquence des vêlages gémellaires, il est envisageable d'accroître par sélection le taux de gémellité chez les bovins de races à viande.

A partir des informations collectées en ferme lors du contrôle national de croissance des bovins, nous avons estimé la fréquence des naissances gémellaires dans les races à viande françaises en fonction du rang de vêlage des mères et de la saison : cette fréquence est environ 7 fois plus élevée en Maine-Anjou (3,8 et 5,3 p. I0o) qu'en Limousine $(0,5-0,8$ p. 100) ; la Charolaise $(3,2-3,4$ p. 100) et la Blonde d'A quitaine (1, I-0,9 p. roo) ont des fréquences intermédiaires. Ces résultats confirment nos précédentes observations où nous avions noté des différences entre régions et une certaine répétabilité entre années du taux de veaux jumeaux des grands élevages. A cette occasion, nous avions aussi constaté que, si la fréquence des couples de jumeaux monozygotes parmi l'ensemble des couples de jumeaux diminue avec l'âge des mères, leur fréquence absolue rapportée à l'ensemble des vêlages reste faible $(0,4 \mathrm{p}$. Ioo) et peu variable avec le rang de mise bas des mères.

Dès 197 I-I 972 nous avons rassemblé dans un même troupeau des vaches ayant eu naturellement 2 à 5 fois des veaux jumeaux. Ces 2 I Maine-Anjou et 18 Charolaises constituent le troupeau de femelles fondatrices que nous avons exploité durant 3 années en race pure, 48 et 41 p. Ioo respectivement de leurs mises bas dans leurs élevages d'origine, avaient donné naissances à des veaux jumeaux.

Après ces 3 années, I 9 couples de jumeaux sont nés (32 p. roo des vêlages). A la suite des premiers vêlages observés dans le troupeau, mais résultant des fécondations dans les élevages d'origine des vaches fondatrices, 44 p. Ioo des Maine-Anjou et I2 p. Ioo des Chavolaises ont engendré des veaux jumeaux. En ne considérant que les fécondations obtenues dans le troupeau, 24 et 36 p. Ioo des vêlages de ces races respectivement ont produit des veaux jumeaux. Parmi les 28 premières fondatrices exploitées durant deux campagnes dans les mêmes conditions, 7 (25 p. 100) ont à nouveau donné au moins une fois des jumeaux. Si nous rassemblons toutes les performances des vaches fondatrices, il ressort alors que 23 p. Ioo d'entre elles ont eu naturellement au moins 4 paires de jumeaux.

Ces premiers résultats sont encourageants. La poursuite de ce travail nécessite, d'une part la recherche de critères précoces prédicteurs de l'aptitude à la gémellité et, d'autre part, le recours aux techniques de superovulation et transplantation d'œufs. C'est dans de telles conditions que nous allons tenter de préciser les modalités d'application d'une sélection efficace sur l'aptitude à la gémellité.

\section{WIRTSCHAFLICHE AUSWIRKUNGEN DER EINKREUZUNG} VON HOLSTEIN-FRIESIANS IN DEUTSCHE SCHWARZBUNTE RINDER

\section{E. ERNST. - Institut für Tierucht und Tierzhaltung, Universität Kiel (BRD).}

Die Einkreuzung von Holstein-Friesians in deutsche schwarzbunte Rinder hat die Ausgangspopulation der deutschen Rinder verändert. $F_{1}$-Tiere und Deutsche Schwarzbunte unterscheiden sich in wichtigen Merkmalen signifikant. $F_{1}$-Tiere übertreffen die deutschen Schwarzbunten im Gewicht um 6 Prozent in der Widerristhöhe um 4 Prozent in der Milchleistung um 13 Prozent 